\title{
The Shortest Path Algorithms of Hypergraphs Based on Search Strategies
}

\author{
Xinquan Chen ${ }^{1,2^{*}}$ \\ ${ }^{1}$ School of Computer Science \& Engineering, Chongqing Three Gorges University, Chongqing, China. \\ 2 Web Sciences Center, University of Electronic Science \& Technologhy of China, Chengdu, China. \\ *Corresponding author. Tel.: 0086-23-58105157; email: chenxqscut@126.com \\ Manuscript submitted March 22, 2014; accepted August 23, 2014.
}

\begin{abstract}
In order to search the shortest path from hypergraphs with unweighted hyperedges, a kind of searching algorithm based on the width-first strategy is presented at first. For hypergraphs with weighted hyperedges, a kind of searching algorithm based on the minimum-cost-first strategy to search the shortest path is presented. In order to enhance the reliability of the two algorithms, their correctness is proven in theory. The ideas and outlines of the two algorithms are illuminated by using two examples. Through the simulation of some artificial hypergraphs, the two algorithms are compared in time cost and the average percentage between the number of nodes in branching trees and the number of all nodes. We find that they can get the same average percentage. This result is consistent with the actual instance. In the end, it gives a research expectation to disinter and popularize the two algorithms.
\end{abstract}

Key words: Hypergraph, minimum-cost-first, shortest path, width-first.

\section{Introduction}

Due to the expansion of application fields, exploring valuable information from some massive, multidimensional, and multiscale objects attracts the attention of some researchers. In exploring different relationships and interesting characters of complex networks, hypergraph model obtains further research and application. Today, hypergraph [1]-[4], which is an extension of the traditional graph theory, gets more attention in the fields of complex network analysis, Internet searching, data mining, and so on. The shortest path of hypergraph can depict the connection degree of two different nodes in the complex network system from one side. So developing effective and efficient algorithms that search the shortest path from hypergraphs has become an important research task.

As we know, Bellman-Ford-Moore algorithm, which searches the shortest paths from a single source node to all of the other nodes in a weighted graph, is the first shortest path algorithm. Almost at the same time, two famous shortest path algorithms of weighted graphs are proposed by Dijkstra and Floyd respectively. Li ChunMing [5] and Gong Qu et al. [6] presented two shortest path algorithms of hypergraphs according to the ideas of searching the shortest paths of graph. This paper presents two more efficient shortest path algorithms of hypergraphs by using two kind of effective search strategies and the methods that constructs the set of associated hyperedges of node, the set of nodes of hyperedge, and the set of associated nodes of node.

The remainder of this paper is organized as follows. Section 2 gives the definition of the shortest path of hypergraphs and some related definitions. Section 3 presents a kind of width-first searching algorithm from 
a hypergraph with unweighted hyperedges and a kind of minimum-cost-first searching algorithm from a hypergraph with weighted hyperedges. Section 4 further explains the two algorithms using two examples. Section 5 compares and validates the two algorithms by simulation experiments. Conclusions and future works are presented in Section 6.

\section{The Definition of the Shortest Path of Hypergraphs}

Suppose a hypergraph $H$ is described by a set of nodes $O S=\left\{o_{1}, o_{2}, \ldots, o_{n}\right\}$, a set of weight of nodes $O W=$ $\left\{o w_{1}, o w_{2}, \ldots, o w_{n}\right\}$, a set of hyperedges $E S=\left\{e_{1}, e_{2}, \ldots, e_{m}\right\}$ that describes the group relationships in the set of nodes $O S$, and a set of weight of hyperedges $E W=\left\{e w_{1}, e w_{2}, \ldots, e w_{m}\right\}$.

Definition 1. Refering to the shortest path concept of graph, in hypergraph $H$, the length of the shortest hyperedge path from node $o_{i}(i=1,2, \ldots, n)$ to another node $o_{j}(j \neq i), \operatorname{SEP}\left(o_{i}, o_{j}\right)$, is defined as:

$$
\operatorname{SEP}\left(o_{i}, o_{j}\right)=\min \left\{\left(e w_{k}+\ldots+e w_{s}\right)\right\}, \quad o_{i} \stackrel{e_{k}}{\longrightarrow} \ldots \stackrel{e_{s}}{\longrightarrow} o_{j}, k, \ldots, s \in\{1,2, \ldots, m\}
$$

where $\left(e_{k}, \ldots, e_{s}\right)$ is a sequence of hyperedges from node $o_{i}$ to node $o_{j}$. The shortest hyperedge path from node $o_{i}$ to node $o_{j}$ is a sequence of hyperedges with the minimal weight sum of hyperedges. Perhaps there are multiple sequences of hyperedges with the minimal weight sum of hyperedges. If there is no sequence of hyperedges from node $o_{i}$ to node $o_{j}$, then the shortest hyperedge path from node $o_{i}$ to node $o_{j}$ does not exist.

Definition 2. Refering to the shortest path concept of graph, in hypergraph $H$, the length of the shortest node path from node $o_{i}(i=1,2, \ldots, n)$ to another node $o_{j}(j \neq i), S N P\left(o_{i}, o_{j}\right)$, is defined as:

$$
\operatorname{SNP}\left(o_{i}, o_{j}\right)=\min \left\{\left(o w_{i}+\ldots+o w_{j}\right)\right\}, \quad o_{i} \stackrel{e_{k}}{\longrightarrow} \ldots \stackrel{e_{s}}{\longrightarrow} o_{j}, k, \ldots, s \in\{1,2, \ldots, m\}
$$

Where $\left(o_{i}, \ldots, o_{j}\right)$ is a sequence of nodes from node $o_{i}$ to node $o_{j}$. The shortest node path from node $o_{i}$ to node $o_{j}$ is a sequence of nodes with the minimal weight sum of nodes. Perhaps there are multiple sequences of nodes with the minimal weight sum of nodes. If there is no sequence of nodes from node $o_{i}$ to node $o_{j}$, then the shortest node path from node $o_{i}$ to node $o_{j}$ does not exist.

Definition 3. The associated matrix [1, 2], $A=\left(a_{i k}\right)_{n^{*} m}$, between the set of nodes, $\left\{o_{1}, o_{2}, \ldots, o_{n}\right\}$, and the set of hyperedges, $\left\{e_{1}, e_{2}, \ldots, e_{m}\right\}$, is defined as:

$$
a_{i k}=\left\{\begin{array}{ll}
1 & o_{i} \in e_{k} \\
0 & o_{i} \notin e_{k}
\end{array}, \quad i=1,2, \ldots, n ; k=1,2, \ldots, m\right.
$$

Definition 4. The set of hyperedges of node $o_{i}(i=1,2, \ldots, n)$ is defined as:

$$
\operatorname{AES}\left(o_{i}\right)=\left\{e_{k} \mid o_{i} \in e_{k}, k \in\{1,2, \ldots, m\}\right\} .
$$

Usually, the number of hyperedges of node $o_{i}$ is regarded as its degree of associated hyperedges, denoted by $\left|\operatorname{AES}\left(o_{i}\right)\right|$.

Definition 5. The set of nodes of hyperedge $e_{k}(k=1,2, \ldots, m)$ is defined as:

$$
\operatorname{EOS}\left(e_{k}\right)=\left\{o_{i} \mid o_{i} \in e_{k}, i \in\{1,2, \ldots, n\}\right\} .
$$

Usually, the number of nodes contained in hyperedge $e_{k}$ is regarded as its degree of associated nodes, 
denoted by $\left|E O S\left(e_{k}\right)\right|$.

Definition 6. The set of associated nodes of node $o_{i}(i=1,2, \ldots, n)$ is defined as:

$$
\operatorname{AOS}\left(o_{i}\right)=\left\{o_{j} \mid\left\{o_{j}, o_{i}\right\} \subseteq e_{k}, j \neq i, j \in\{1,2, \ldots, n\} ; k \in\{1,2, \ldots, m\}\right\}
$$

Usually, the number of nodes shared with node $o_{i}$ in common hyperedges is regarded as its degree of straight-connected nodes, denoted by $\left|A O S\left(o_{i}\right)\right|$.

Definition 7. The cost function $f\left(o_{i}\right)$ of node $o_{i}(i=1,2, \ldots, n)$ in its any expanding tree is defined as:

If $o_{i}$ is the root of the expanding tree, set $f\left(o_{i}\right)=0$;

If $o_{i}$ is not the root of the expanding tree, set

$$
f\left(o_{i}\right)=f\left(\operatorname{parent}\left(o_{i}\right)\right)+e w\left(\operatorname{MinEdge}\left(\operatorname{parent}\left(o_{i}\right), o_{i}\right)\right)
$$

where parent $\left(o_{i}\right)$ is the father node of node $o_{i}$, MinEdge(parent $\left.\left(o_{i}\right), o_{i}\right)$ is the hyperedge with minimum weight in the set of hyperedges that contains node parent $\left(o_{i}\right)$ and node $o_{i}$, and $\operatorname{ew}\left(\operatorname{MinEdge}\left(\operatorname{parent}\left(o_{i}\right), o_{i}\right)\right)$ is the weight of hyperedge MinEdge(parent $\left.\left(o_{i}\right), o_{i}\right)$. Obviously, this is a recursive definition.

\section{The Shortest Path Algorithms of Hypergraphs Based on Search Strategies}

Because the shortest node path algorithms of hypergraphs is similar to the shortest hyperedge path algorithms of hypergraphs, so we only discuss the latter.

\subsection{A Width-First Searching Algorithm of Hypergraphs with Unweighted Hyperedges}

The width-first searching algorithm of hypergraphs with unweighted hyperedges is presented below.

Algorithm Name: a width-first searching algorithm of hypergraphs with unweighted hyperedges (Algorithm 1).

Input: a set of nodes, $O S=\left\{o_{1}, o_{2}, \ldots, o_{n}\right\}$; a set of hyperedges, $E S=\left\{e_{1}, e_{2}, \ldots, e_{m}\right\}$; a associated matrix, $A=$ $\left(a_{i k}\right)_{n^{*} m}$, between the set of nodes and the set of hyperedges.

Output: the shortest hyperedge path between a pair of nodes and its length.

\section{Procedure:}

Step1. According to the matrix $A$, definition 4, and definition 6, construct two sets $A E S\left(o_{i}\right)$ and $A O S\left(o_{i}\right)$ for every node $o_{i}(i=1,2, \ldots, n)$. According to the matrix $A$ and definition 5 , construct the set $\operatorname{EOS}\left(e_{k}\right)$ for every hyperedge $e_{k}(k=1,2, \ldots, m)$.

The set $A E S\left(o_{i}\right)$ of node $o_{i}$ and the set $\operatorname{EOS}\left(e_{k}\right)$ of hyperedge $e_{k}$ can be constructed easily from the matrix $A$. The set $\operatorname{AOS}\left(o_{i}\right)$ is constructed from two sets $\operatorname{AES}\left(o_{i}\right)$ and $\operatorname{EOS}\left(e_{k}\right)$. The concrete constructing procedure is described as follows:

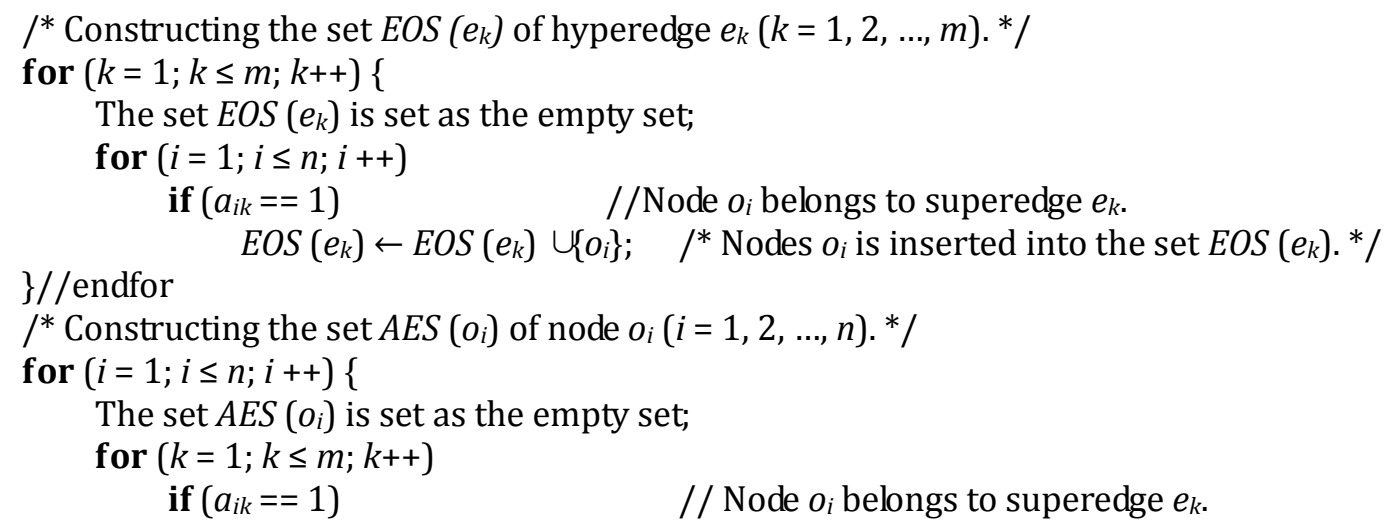


\}//endfor

$$
A E S\left(o_{i}\right) \leftarrow A E S\left(o_{i}\right) \cup\left\{e_{k}\right\} ; \quad / * \text { Superedge } e_{k} \text { is inserted into the set } A E S\left(o_{i}\right) . * /
$$

$/{ }^{*}$ Constructing the set $\operatorname{AOS}\left(o_{i}\right)$ of node $o_{i}(i=1,2, \ldots, n) . * /$

for $(i=1 ; i \leq n ; i++)\{$

The set $A O S\left(o_{i}\right)$ is set as the empty set;

for (every element $e_{k}$ in the set AES $\left.\left(o_{i}\right)\right) \quad / *$ Superedge $e_{k}$ belongs to the set $A E S\left(o_{i}\right) . * /$ $A O S\left(o_{i}\right) \leftarrow A O S\left(o_{i}\right) \cup\left(E O S\left(e_{k}\right)-\left\{o_{i}\right\}\right) ; \quad /^{*}$ The other elements except node $o_{i}$ in the set EOS

$\left(e_{k}\right)$ is inserted into the set $A O S\left(o_{i}\right) . * /$

\}//endfor

Step 2. According to the set $A O S\left(o_{i}\right)$ of node $o_{i}(i=1,2, \ldots, n)$, the width-first searching strategy is used to construct the branching tree of node $o_{i}$, whose root is node $o_{i}$. The concrete constructing procedure is described as follows:

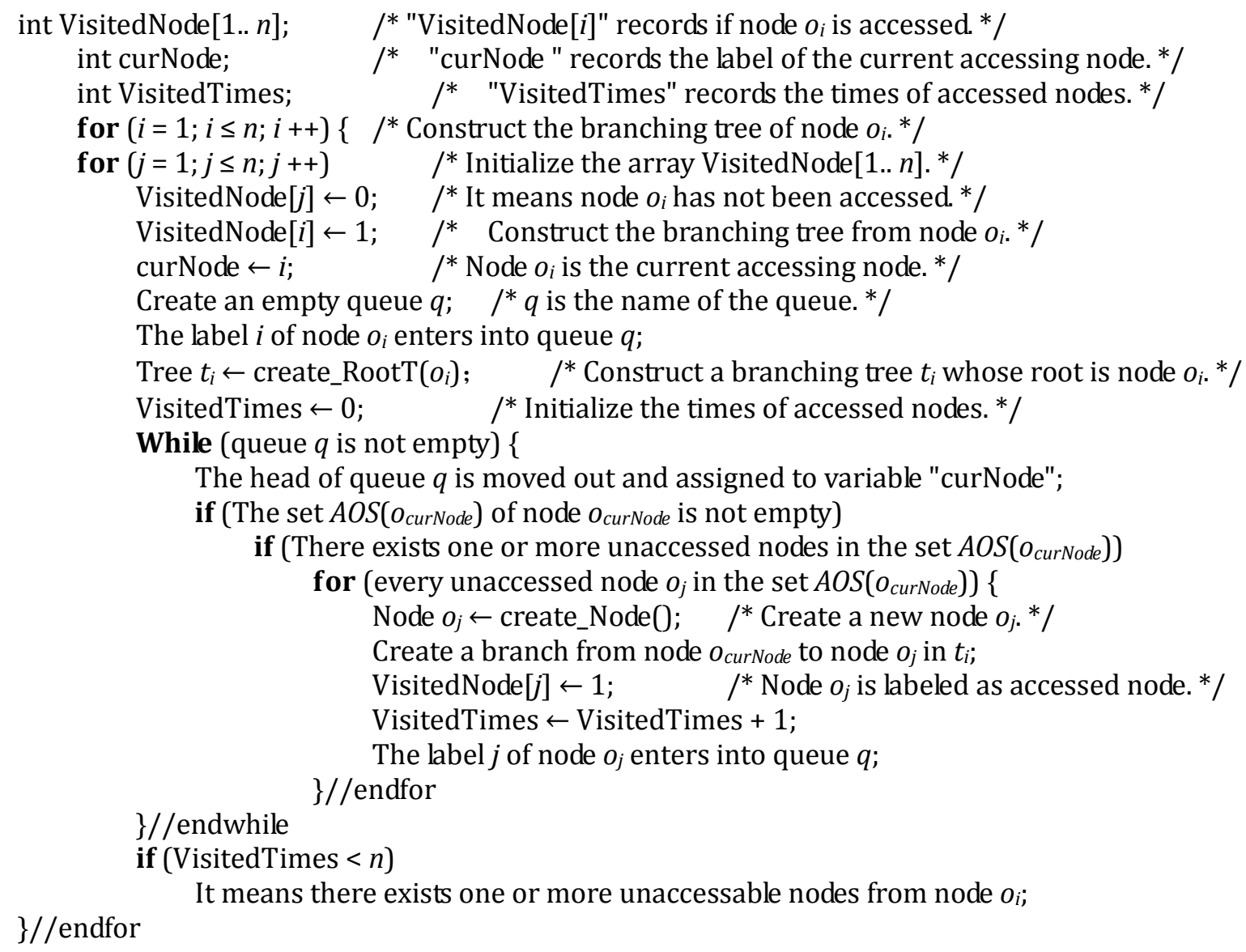

Step 3. The path from the root node $o_{i}$ to another node in the branch tree $t_{i}(i=1,2, \ldots, n)$ is its shortest path. And the height of the accessed node is the length of its shortest path.

\subsection{A minimum-Cost-First Searching Algorithm of Hypergraphs with Weighted Hyperedges}

The minimum-cost-first searching algorithm of hypergraphs with unweighted hyperedges is presented below.

Algorithm Name: a minimum-cost-first searching algorithm of hypergraphs with weighted hyperedges (Algorithm 2).

Input: a set of nodes, $O S=\left\{o_{1}, o_{2}, \ldots, o_{n}\right\}$; a set of hyperedges, $E S=\left\{e_{1}, e_{2}, \ldots, e_{m}\right\}$; a set of weight of hyperedges, $E W=\left\{e w_{1}, e w_{2}, \ldots, e w_{m}\right\}$; a associated matrix, $A=\left(a_{i k}\right)_{n^{*} m}$, between the set of nodes and the set of hyperedges. 
Output: the shortest hyperedge path between a pair of nodes and its length.

\section{Procedure:}

Step 1. The same as the Step1 of Algorithm 1.

Step 2. If the set $\operatorname{AOS}\left(o_{i}\right)$ of node $o_{i}(i=1,2, \ldots, n)$ is not empty, then create an array AssMEW $\left(o_{i}\right)[1$.. $\left.\left|A O S\left(o_{i}\right)\right|\right]$ that stores its associated nodes, hyperedges with minimum weight between node $o_{i}$ and its associated nodes, and the weights of the hyperedges.

For example, suppose node $o_{j}$ is an associated node of node $o_{i}$, then a structure $\operatorname{Ass} M E W\left(o_{i}\right)[j]=<o_{j}$, $\operatorname{MinEdge}\left(o_{i}, o_{j}\right)$, ew $\left(\operatorname{MinEdge}\left(o_{i}, o_{j}\right)\right)>$ can be designed. Where $\operatorname{MinEdge}\left(o_{i}, o_{j}\right)$ is a hyperedge with minimum weight that contains two associated nodes $o_{i}$ and $o_{j}$, and $\operatorname{ew}\left(\operatorname{MinEdge}\left(o_{i}, o_{j}\right)\right)$ is the weight of the hyperedge MinEdge $\left(o_{i}, o_{j}\right)$.

An implementation procedure of this step is described as follows:

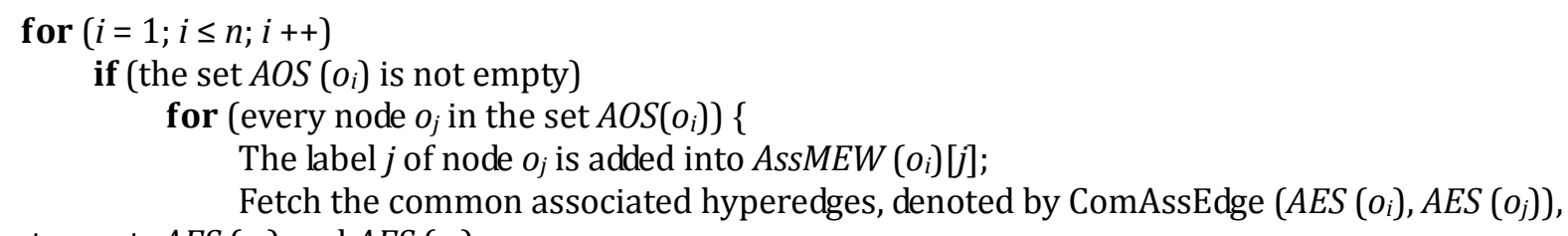
from two sets $A E S\left(o_{i}\right)$ and $A E S\left(o_{j}\right)$;

for (every hyperedge in the set ComAssEdge $\left(\operatorname{AES}\left(o_{i}\right), \operatorname{AES}\left(o_{j}\right)\right)$ ) \}//endfor $\operatorname{AssMEW}\left(o_{i}\right)[j]$ always records the hyperedge with minimum weight and its weight;

Step 3. According to the set $A O S\left(o_{i}\right)$ of node $o_{i}(i=1,2, \ldots, n)$, the minimum-cost-first searching strategy is used to construct the branching tree of node $o_{i}$, whose root is node $o_{i}$. When constructing the branching tree, the cost function of every node is computed according to Definition 7 . The concrete constructing procedure is described as follows:

int curNode;

int CanVisitNode[1..n];

bool ExistExpandNode; in the branching tree. */
$/ * \quad$ "curNode " records the label of the current accessing node. */

/* "CanVisitNode [i]" records if node $o_{i}$ can be accessed. */

$/ *$ "ExistExpandNode " records if there exists one or more expandable nodes

for $(i=1 ; i \leq n ; i++)$

$/{ }^{*}$ Construct the branching tree of node $o_{i \cdot} * /$

for $(j=1 ; j \leq n ; j++)\{$

CanVisitNode $[j] \leftarrow 1 ; \quad \quad / *$ It means node $o_{j}$ can be accessed. */

$g\left(o_{j}\right) \leftarrow+\infty ; \quad \quad / *$ Initialize the preference-selecting function $g\left(o_{j}\right) . * /$

\}$/ /$ endfor

$f\left(o_{i}\right) \leftarrow 0 ; \quad \quad / *$ The cost function $f\left(o_{i}\right)$ of root $o_{i}$ is assigned by zero. */

$g\left(o_{i}\right) \leftarrow f\left(o_{i}\right) ; \quad \quad / *$ The preference-selecting function $g\left(o_{i}\right)$ of root $o_{i}$ is updated by $f\left(o_{i}\right) . * /$

curNode $\leftarrow i ; \quad / *$ Node $o_{i}$ is the current accessing node. $* /$

CanVisitNode $[i] \leftarrow 0 ; \quad / *$ Node $o_{i}$ cannot be accessed after expanded. *

Tree $t_{i} \leftarrow$ create_RootT $\left(o_{i}\right) ; \quad /{ }^{*}$ Construct a branching tree $t_{i}$ whose root is node $o_{i}{ }^{*} /$

do \{

if (the set $A O S\left(o_{\text {curNode }}\right)$ is empty, or node $o_{\text {curNode }}$ cannot be expanded) \{

Node $o_{\text {curNode }}$ is labeled as a terminal leaf node;

\}

CanVisitNode[curNode] $\leftarrow 0 ; \quad / *$ It means node $o_{\text {curNode }}$ cannot be expanded. */

else \{

for (every accessable node $o_{j}$ in the set $A O S\left(o_{\text {curNode }}\right)$ ) \{

Node $o_{\text {curNode }}$ is regarded as the parent node of node $o_{j}$, and the cost function $f\left(o_{j}\right)$ of

node $o_{j}$ is computed by equation (7);

if $\left.f\left(o_{j}\right)<g\left(o_{j}\right)\right)\{$

if (node $o_{j}$ is already in the branching tree $t_{i}$ )

Delete the old branch reaching node $o_{j}$ in the branching tree $t_{i}$; 
updated by $f\left(o_{j}\right) . * /$

else

Node $o_{j} \leftarrow$ create_Node(); $\quad / *$ Create a new node $o_{j} *$ /

Insert a new branch from node $o_{\text {curNode }}$ to node $o_{j}$ in the branching tree $t_{i}$;

$g\left(o_{j}\right) \leftarrow f\left(o_{j}\right) ; \quad \quad / *$ The preference-selecting function $g\left(o_{j}\right)$ of node $o_{j}$ is

\}$/ /$ endif

\} //endfor

been expanded. */

Node $o_{\text {curNode }}$ is labeled as a nonleaf node that cannot be expanded; $\quad / *$ Node $o_{\text {curNode }}$ has

\}$/ /$ endif

CanVisitNode[curNode] $\leftarrow 0 ; \quad \quad / *$ Node $o_{\text {curNode }}$ cannot be expanded anymore. */

ExistExpandNode $\leftarrow$ false;

if (There exists one or more accessable nodes in the branching tree $t_{i}$ ) /* There exists

one or more nonleaf nodes in the branching tree $t_{i}{ }^{*} /$

ExistExpandNode $\leftarrow$ true;

if (ExistExpandNode)

The label of the nonleaf node that gets the minimum value of preference-selecting function

in the branching tree $t_{i}$ is assigned to "curNode"; $\quad / *$ Choose the node with minimum value of

preference-selecting function from those elements whose value is equal to one in array CanVisitNode[1.. $n]$. $* /$

\} while (ExistExpandNode);

if $($ VisitedTimes $<n$ )

\}//endfor

It means there exists one or more unaccessable nodes from node $o_{i}$;

Step 4. The path from the root node $o_{i}$ to another node in the branch tree $t_{i}(i=1,2, \ldots, n)$ is its shortest path. And the value of the cost function of the accessed node is the length of its shortest path.

\subsection{Some Notes of Two Algorithms}

The minimum-cost-first searching algorithm of hypergraphs with unweighted hyperedges is presented below.

Some notes of Algorithm 1 are listed below:

1) In Step 1, the ordered (in ascending order) adjacency list [3], [4], which stores the set of associated hyperedges of node, the set of nodes of hyperedge, and the set of associated nodes of node, is similar to the adjacency list structure of graph.

2) In Step 2, the rule, "from top to bottom, from left to right", is used to create the branch tree $t_{i}$. Here, queue structure can effectively implement this creating order of nodes based on this rule, which reflects the wide-first searching strategy.

3) Time and space complexity analysis of Algorithm 1:

Step 1 needs Time $=O\left(n \cdot m+\sum_{i=1}^{n} \sum_{e_{k} \in A E S\left(O_{i}\right)}\left(\left|\operatorname{EOS}\left(e_{k}\right)\right| \cdot\left|A O S\left(O_{i}\right)\right|\right)\right)$ and Space $=O\left(n \cdot m+\sum_{i=1}^{n}\left|A E S\left(o_{i}\right)\right|+\right.$ $\left.\sum_{i=1}^{n}\left|\operatorname{AOS}\left(o_{i}\right)\right|+\sum_{k=1}^{m}\left|\operatorname{EOS}\left(e_{k}\right)\right|\right)$

Step 2 needs Time $=O\left(n^{2}\right)$ and Space $=O\left(n^{2}+\sum_{i=1}^{n}\left|A O S\left(o_{i}\right)\right|\right)$.

Step 3 needs Time $=O\left(n^{2}\right)$ and Space $=O\left(n^{2}\right)$.

4) This is a kind of searching algorithm based on the width-first strategy. It searches the shortest path from a hypergraph with unweighted hyperedges by constructing the set of associated hyperedges of every node, the set of nodes of every hyperedge, and the set of associated nodes of every node.

Some notes of Algorithm 2 are listed below: 
1) If Step1 uses the improving technique used in Step2, then the procedure of Step1, Constructing the set $A O S\left(o_{i}\right)$ of node $o_{i}(i=1,2, \ldots, n)$, can be replaced by the following procedure.

$/{ }^{*}$ Create $\operatorname{AssMEW}\left(o_{i}\right)$ that is implemented by the array AssMEW $\left(o_{i}\right)\left[1 . .\left|A O S\left(o_{i}\right)\right|\right] . * /$

for $(i=1 ; i \leq n ; i++)\{$

$\operatorname{AssMEW}\left(o_{i}\right)$ is set as the empty set;

$* /$

for (every element $e_{k}$ in the set $\left.A E S\left(o_{i}\right)\right) \quad / *$ Superedge $e_{k}$ belongs to the set $A E S\left(o_{i}\right)$ of node $o_{i}$.

$\operatorname{AssMEW}\left(o_{i}\right) . * /$

for (every node $o_{j}$ in the set $\operatorname{EOS}\left(e_{k}\right)$ )

if (node $o_{j}$ and node $o_{i}$ are different)

if (node $o_{j}$ is not in AssMEW(o $\left.o_{i}\right)$ )

${ }^{*}$ Node $o_{j}$, hyperedge $e_{k}$, and the weight of hyperedge $e_{k}$ are inserted into

$\left.\operatorname{AssMEW}\left(o_{i}\right)\right)$

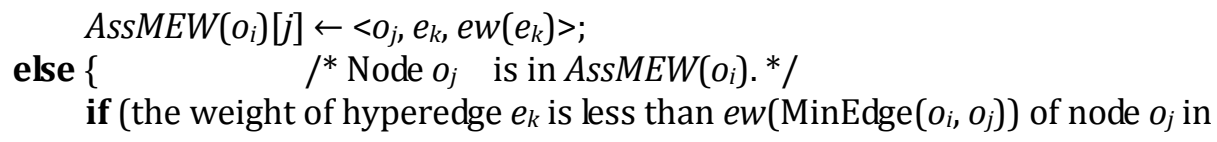

latter two items of node $o_{j}$ in $\operatorname{AssMEW(o_{i}).*/}$

$I^{*}$ Hyperedge $e_{k}$ and the weight of hyperedge $e_{k}$ are used to update the

$\operatorname{AssMEW}\left(o_{i}\right)[j] \leftarrow<o_{j}, e_{k}, e w\left(e_{k}\right)>$;

\}//endfor

2) In Step 3, "nonleaf nodes" are those nodes that can be expanded in the current branching tree. If the value of $f$ function, which is computed by using equation (7), of every associated node of node $o_{\text {curNode }}$ is larger than or equal to the value of $g$ function of the associated node, then "node $o_{\text {curNode }}$ cannot be expanded".

3) Time and space complexity analysis of Algorithm 2:

In Step 1, it needs the same time and space with Step 1 of Algorithm 1.

Step 2 needs Time $\left.=O\left(\sum_{i=1}^{n}\left(\left|A O S\left(o_{i}\right)\right|+\sum_{o_{j} \in A O S\left(o_{i}\right)}\left(\left|A E S\left(o_{i}\right)\right|+\left|A E S\left(o_{j}\right)\right|\right)\right)\right)\right)$ and Space $=O\left(n+m+\sum_{i=1}^{n}\left|A E S\left(o_{i}\right)\right|\right.$

$\left.+\sum_{i=1}^{n}\left|\operatorname{AOS}\left(o_{i}\right)\right|\right)$

Step 3 needs Time $=O\left(n^{2}\right)$ and Space $=O\left(n^{2}\right)$.

Step 4 needs Time $=\theta\left(n^{2}\right) \sim O\left(n \cdot \sum_{i=1}^{n}\left|A O S\left(o_{i}\right)\right|\right)$ and Space $=O\left(n^{2}+\sum_{i=1}^{n}\left|A O S\left(o_{i}\right)\right|\right)$.

4) This is a kind of searching algorithm based on the minimum-cost-first strategy. It searches the shortest path from a hypergraph with weighted hyperedges by constructing the set of associated hyperedges of every node, the set of nodes of every hyperedge, and the set of associated nodes with minimum hyperedge and its weight of every node.

Table 1. The Time Complexity of Three Algorithms

\begin{tabular}{cccc}
\hline & Algorithms from [5] & $\begin{array}{c}\text { Algorithms } \\
\text { from [6] }\end{array}$ & Algorithms from This paper \\
\hline $\begin{array}{c}\text { Unweighted } \\
\text { hyperedges }\end{array}$ & $O\left(n \cdot m \cdot\left(\max _{i=1,2, \ldots, m}\left|e_{i}\right|\right)^{2}\right)$ & $O\left(n^{3}\right)$ & $O\left(n \cdot m+\sum_{i=1}^{n} \sum_{e_{k} \in A E S\left(O_{i}\right)}\left(\left|\operatorname{EOS}\left(e_{k}\right)\right| \cdot\left|\operatorname{AOS}\left(O_{i}\right)\right|\right)\right)$ \\
\hline $\begin{array}{c}\text { Weighted } \\
\text { hyperedges }\end{array}$ & $O\left(n \cdot m \cdot\left(\max _{i=1,2, \ldots m}\left|e_{i}\right|\right)^{2}+n^{3}\right)$ & $O\left(n^{3}\right)$ & $O\left(n \cdot m+\sum_{i=1}^{n} \sum_{e_{k} \in A E S S\left(O_{i}\right)}\left(\left|\operatorname{EOS}\left(e_{k}\right)\right| \cdot\left|\operatorname{AOS}\left(O_{i}\right)\right|\right)+n \cdot \sum_{i=1}^{n}\left|A O S\left(O_{i}\right)\right|\right)$ \\
\hline
\end{tabular}




\subsection{Comparison with Two Other Algorithms}

Table 1 gives the comparison results the algorithms from this paper with other algorithms [5], [6] in time complexity. In some cases, our algorithms can be more efficient by constructing the set of associated hyperedges of node, the set of nodes of hyperedge, and the set of associated nodes of node.

\subsection{The Proof of Correctness of Two Algorithms}

Theorem 1. In a hypergraph with unweighted hyperedges, when constructing a branching tree for every node using a kind of searching algorithm based on the width-first strategy, the path from the root to another node in the branching tree is its one shortest path, the depth of every node in the branching tree is the length of the shortest path from the root to the node.

Proof: The conclusion is obvious because of the expanding strategy of nodes in branching trees. In the constructing procedure of branching trees, after expanded a node, its successor node is chosen to expand according to the rule, "from top to bottom, from left to right". The branching trees contain the paths from the root to other nodes.

Theorem 2. In a hypergraph with weighted hyperedges, when constructing a branching tree for every node using a kind of searching algorithm based on the minimum-cost-first strategy, the path from the root to another node in the branching tree is its one shortest path, the value of preference-selecting function of every node in the branching tree is the length of the shortest path from the root to the node.

Proof: The conclusion is also obvious because of the expanding strategy of nodes in branching trees. In each node expansion of the branching trees, the nonleaf node with the minimum value of preference-selecting function is chosen to expand. The branching trees also contain the paths from the root to other nodes.

\section{Two Examples to Explain the Algorithms}

\subsection{Example 1}

Suppose there is a hypergraph that is described by a set of nodes $\left\{o_{1}, o_{2}, o_{3}, o_{4}, o_{5}\right\}$ and a set of hyperedges $\left\{e_{1}=\left\{o_{1}, o_{2}, o_{3}\right\}, e_{2}=\left\{o_{2}, o_{3}\right\}, e_{3}=\left\{o_{4}, o_{5}\right\}\right\}$.

The results of example 1 after executed Step 1 of Algorithm 1 are given in Table 2 and Table 3.

Table 2. The Sets AES and AOS of Nodes in Algorithm 1

\begin{tabular}{llllll}
\hline $\mathrm{O}_{\mathrm{i}}$ & $\mathrm{o}_{1}$ & $\mathrm{o}_{2}$ & $\mathrm{o}_{3}$ & $\mathrm{o}_{4}$ & $\mathrm{o}_{5}$ \\
\hline $\operatorname{AES}\left(\mathrm{O}_{\mathrm{i}}\right)$ & $\left\{\mathrm{e}_{1}\right\}$ & $\left\{\mathrm{e}_{1}, \mathrm{e}_{2}\right\}$ & $\left\{\mathrm{e}_{1}, \mathrm{e}_{2}\right\}$ & $\left\{\mathrm{e}_{3}\right\}$ & $\left\{\mathrm{e}_{3}\right\}$ \\
\hline $\operatorname{AOS}\left(\mathrm{o}_{\mathrm{i}}\right)$ & $\left\{\mathrm{o}_{2}, \mathrm{o}_{3}\right\}$ & $\left\{\mathrm{o}_{1}, \mathrm{O}_{3}\right\}$ & $\left\{\mathrm{o}_{1}, \mathrm{o}_{2}\right\}$ & $\left\{\mathrm{o}_{5}\right\}$ & $\left\{\mathrm{o}_{4}\right\}$ \\
\hline
\end{tabular}

Table 3. The Set EOS of Hyperedges in Algorithm 1

\begin{tabular}{llll}
\hline $\mathrm{e}_{\mathrm{k}}$ & $\mathrm{e}_{1}$ & $\mathrm{e}_{2}$ & $\mathrm{e}_{3}$ \\
\hline $\mathrm{EOS}\left(\mathrm{e}_{\mathrm{k}}\right)$ & $\left\{\mathrm{0}_{1}, \mathrm{O}_{2}, \mathrm{O}_{3}\right\}$ & $\left\{\mathrm{o}_{2}, \mathrm{O}_{3}\right\}$ & $\left\{\mathrm{O}_{4}, \mathrm{O}_{5}\right\}$ \\
\hline
\end{tabular}

The results of example 1 after executed Step 2 of Algorithm 1 are given in Fig. 1.
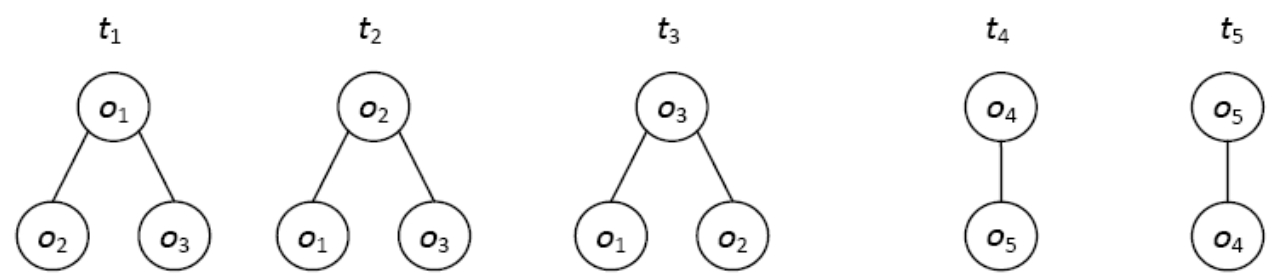

Fig. 1. The branching trees of five nodes in Algorithm 1. 
The results of example 1 after executed Step 1 of Algorithm 2 are the same as Algorithm 1 except that the set $A O S\left(o_{i}\right)$ in Algorithm 2 contains more information, which is listed in Table 4.

Table 4. The Set AOS with Minimum Hyperedge of Nodes in Algorithm 2

\begin{tabular}{ll}
\hline$o_{i}$ & $A O S\left(o_{i}\right)$ \\
\hline$o_{1}$ & $\left\{<O_{2}, e_{1}, 2>,<O_{3}, e_{1}, 2>\right\}$ \\
\hline$o_{2}$ & $\left\{<O_{1}, e_{1}, 2>,<O_{3}, e_{2}, 1>\right\}$ \\
\hline$o_{3}$ & $\left\{<O_{1}, e_{1}, 2>,<O_{2}, e_{2}, 1>\right\}$ \\
\hline$o_{4}$ & $\left\{<O_{5}, e_{3}, 1.5>\right\}$ \\
\hline$o_{5}$ & $\left\{<O_{4}, e_{3}, 1.5>\right\}$ \\
\hline
\end{tabular}

In this simple example, the branching trees of five nodes after executed Step 2 of Algorithm 2 are the same as Algorithm 1, although their searching strategies are different.

\subsection{Example 2}

Fig. 2 [7] is a hypergraph that is described by a set of nodes $\left\{v_{1}, v_{2}, v_{3}, v_{4}, v_{5}, v_{6}, v_{7}\right\}$ and a set of hyperedges $\left\{e_{1}=\left\{v_{1}, v_{2}, v_{3}\right\}, e_{2}=\left\{v_{2}, v_{3}\right\}, e_{3}=\left\{v_{3}, v_{5}, v_{6}\right\}, e_{4}=\left\{v_{4}\right\}\right.$. In Algorithm 1 the weights of hyperedges are set as 1. And in Algorithm 2 the weights of hyperedges are set as $\{3,2,3,1\}$.

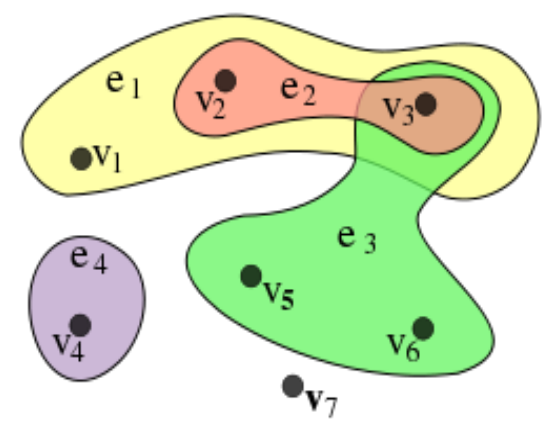

Fig. 2. A hypergraph from Wikipedia [7].

The results of example 2 after executed Step 1 of Algorithm 1 are given in Table 5 and Table 6 .

Table 5. The Sets AES and AOS of Nodes in Algorithm 1

\begin{tabular}{llllllll}
\hline$v_{i}$ & $v_{1}$ & $v_{2}$ & $v_{3}$ & $v_{4}$ & $v_{5}$ & $v_{6}$ & $v_{7}$ \\
\hline$A E S\left(v_{i}\right)$ & $\left\{e_{1}\right\}$ & $\left\{e_{1}, e_{2}\right\}$ & $\left\{e_{1}, e_{2}, e_{3}\right\}$ & $\left\{e_{4}\right\}$ & $\left\{e_{3}\right\}$ & $\left\{e_{3}\right\}$ & $\Phi$ \\
\hline$A O S\left(v_{i}\right)$ & $\left\{v_{2}, v_{3}\right\}$ & $\left\{v_{1}, v_{3}\right\}$ & $\left\{v_{1}, v_{2}, v_{5}, v_{6}\right\}$ & $\Phi$ & $\left\{v_{3}, v_{6}\right\}$ & $\left\{v_{3}, v_{5}\right\}$ & $\Phi$ \\
\hline
\end{tabular}

Table 6. The Set EOS of Hyperedges in Algorithm 1

\begin{tabular}{lllll}
\hline$e_{k}$ & $e_{1}$ & $e_{2}$ & $e_{3}$ & $e_{4}$ \\
\hline $\operatorname{EOS}\left(e_{k}\right)$ & $\left\{v_{1}, v_{2}, v_{3}\right\}$ & $\left\{v_{2}, v_{3}\right\}$ & $\left\{v_{3}, v_{5}, v_{6}\right\}$ & $\left\{v_{4}\right\}$ \\
\hline
\end{tabular}

The results of example 2 after executed Step 2 of Algorithm 1 are given in Fig. 3.

The results of example 2 after executed Step1 of Algorithm 2 are the same as Algorithm 1 except that the set $A O S\left(o_{i}\right)$ in Algorithm 2 contains more information, which is listed in Table 7.

In this simple example, the branching trees of seven nodes after executed Step2 of Algorithm 2 are the same as Algorithm 1, although their searching strategies are different.

If the set of hyperedges in this hypergraph is revised as $\left\{e_{1}=\left\{v_{1}, v_{2}, v_{3}\right\}, e_{2}=\left\{v_{2}, v_{3}\right\}, e_{3}=\left\{v_{3}, v_{5}, v_{6}\right\}, e_{4}=\right.$ $\left.\left\{v_{1}, v_{3}\right\}\right\}$, and the set of weights of hyperedges in Algorithm 2 is revised as $\{3,2,3,0.5\}$, then the branching tree $t_{2}$ of node $v_{2}$ after Step 2 in Algorithm 2 is different from Algorithm 1. 


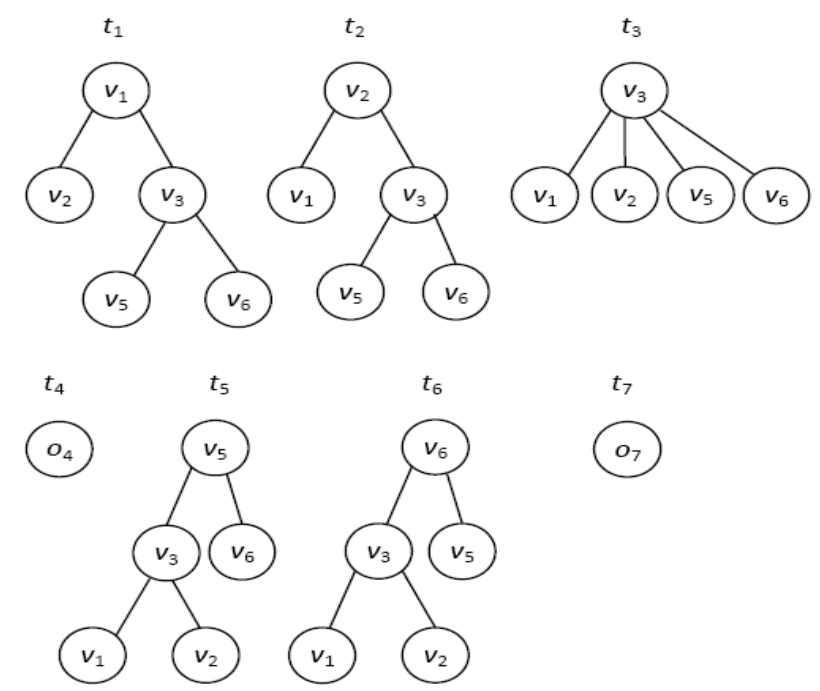

Fig. 3. The branching trees of seven nodes in Algorithm 1.

Table 7. The Set $A O S$ with Minimum Hyperedge of Nodes in Algorithm 2

\begin{tabular}{ll}
\hline$v_{i}$ & $A O S\left(v_{i}\right)$ \\
\hline$v_{1}$ & $\left\{<v_{2}, e_{1}, 3>,<v_{3}, e_{1}, 3>\right\}$ \\
\hline$v_{2}$ & $\left\{<v_{1}, e_{1}, 3>,<v_{3}, e_{2}, 2>\right\}$ \\
\hline$v_{3}$ & $\left\{<v_{1}, e_{1}, 3>,<v_{2}, e_{2}, 2>,<v_{5}, e_{3}, 3>,<v_{6}, e_{3}, 3>\right\}$ \\
\hline$v_{4}$ & $\Phi$ \\
\hline$v_{5}$ & $\left\{<v_{3}, e_{3}, 3>,<v_{6}, e_{3}, 3>\right\}$ \\
\hline$v_{6}$ & $\left\{<v_{3}, e_{3}, 3>,<v_{5}, e_{3}, 3>\right\}$ \\
\hline$v_{7}$ & $\Phi$ \\
\hline
\end{tabular}

\section{Simulation Experiments}

\subsection{Experimental Design}

Our experiments are finished in a personal computer (Intel(R) Pentium(R) Dual CPU T4500 2.3GHz, 2G Memory). Experimental programs are developed using Visual $\mathrm{C}++6.0$ under Windows XP.

To verify the correctness and the validity of Algorithm 1 and Algorithm 2, there will be some experiments of a kind of artificial hypergraph in the next subsection.

The artificial hypergraph, which was used in [8], has $n$ nodes and $m$ hyperedges. The matrix $A=\left(a_{i k}\right)_{n^{*} m}$ is designed by producing $n^{*} m$ random real numbers in the region $[0,1]$ such that 1 is assigned to the elment $a_{i k}$ if the $i^{*} k$-th random real number is larger than threshold parameter $\Delta$, otherwise 0 is assigned to the elment $a_{i k}$. The set $E W=\left\{e w_{1}, e w_{2}, \ldots, e w_{m}\right\}$ is designed by producing $m$ random positive real numbers.

In subsection 5.2, Algorithm 2 will be compared with Algorithm 1 in time cost (measured by second) and property of branching trees by using several hypergraphs with different $n$ and $m$.

Performance of algorithms is measured by time cost (label: ST). A result of algorithms is measured by the average percentage between the number of nodes in branching trees and the number of all nodes (label: Per).

$$
\operatorname{Per}=\frac{1}{n} \sum_{i=1}^{n} \frac{\left|\operatorname{tree}\left(O_{i}\right)\right|}{n}
$$

where $\mid$ tree $\left(O_{i}\right) \mid(i=1,2, \ldots, n)$ is the number of nodes in the branching tree $t_{i}$ whose root is node $O_{i}$. The average percentage can measure the connectivity of a hypergraph. The larger the index is, the better the connectivity of the hypergraph has. 


\subsection{Experimental Results}

Table 8 and Table 9 list some comparative experimental results of Algorithm 1 and Algorithm 2 by processing the artificial hypergraphs with several pairs of parameters $(n, m)$. Label $S T$ means spend time.

Table 8. Comparative Experimental Results of Two Algorithms (Threshold Parameter $\Delta=0.95$ in the Artificial Hypergraphs)

\begin{tabular}{llllll}
\hline \multicolumn{2}{l}{ Pairs of parameters $(n, m)$} & \multicolumn{2}{l}{ Algorithm 1} & \multicolumn{2}{l}{ Algorithm 2} \\
\hline $\boldsymbol{n}$ & $\boldsymbol{m}$ & $\boldsymbol{S T}$ & $\boldsymbol{P e r}(\%)$ & $\boldsymbol{S}$ T & Per(\%) \\
\hline 100 & 5 & 0 & $\mathbf{1 . 9 8}$ & 0 & $\mathbf{1 . 9 8}$ \\
\hline 100 & 10 & 0 & $\mathbf{4 . 4 6}$ & 0 & $\mathbf{4 . 4 6}$ \\
\hline 100 & 20 & 0 & $\mathbf{3 2 . 9 4}$ & 0 & $\mathbf{3 2 . 9 4}$ \\
\hline 100 & 30 & 0 & $\mathbf{6 5 . 8}$ & 0 & $\mathbf{6 5 . 8}$ \\
\hline 100 & 40 & 0 & $\mathbf{7 4 . 1}$ & 0 & $\mathbf{7 4 . 1}$ \\
\hline 100 & 50 & 0 & $\mathbf{8 2 . 9 2}$ & 0 & $\mathbf{8 2 . 9 2}$ \\
\hline 100 & 60 & 0 & $\mathbf{9 0 . 3}$ & 0 & $\mathbf{9 0 . 3}$ \\
\hline 100 & 70 & 0 & $\mathbf{9 6 . 0 7}$ & 0 & $\mathbf{9 6 . 0 7}$ \\
\hline 100 & 80 & 0 & $\mathbf{9 9 . 0 1}$ & 0 & $\mathbf{9 9 . 0 1}$ \\
\hline 100 & 90 & 0 & $\mathbf{1 0 0}$ & 0 & $\mathbf{1 0 0}$ \\
\hline 200 & 5 & 0 & $\mathbf{1 . 7 3}$ & 0 & $\mathbf{1 . 7 3}$ \\
\hline 200 & 10 & 0 & $\mathbf{1 4 . 7 5}$ & 0 & $\mathbf{1 4 . 7 5}$ \\
\hline 200 & 20 & 0 & $\mathbf{3 9 . 2 5}$ & 0 & $\mathbf{3 9 . 2 5}$ \\
\hline 200 & 30 & 0 & $\mathbf{6 4 . 1}$ & 0 & $\mathbf{6 4 . 1}$ \\
\hline 200 & 40 & 0 & $\mathbf{7 3 . 1 8}$ & 0 & $\mathbf{7 3 . 1 8}$ \\
\hline 200 & 50 & 0 & $\mathbf{8 4 . 6 8}$ & 0 & $\mathbf{8 4 . 6 8}$ \\
\hline 200 & 60 & 0 & $\mathbf{9 0 . 2 8}$ & 0 & $\mathbf{9 0 . 2 8}$ \\
\hline 200 & 70 & 0 & $\mathbf{9 5 . 0 8}$ & 0 & $\mathbf{9 5 . 0 8}$ \\
\hline 200 & 80 & 0 & $\mathbf{9 8 . 0 2}$ & 0 & $\mathbf{9 8 . 0 2}$ \\
\hline 200 & 90 & 0 & $\mathbf{1 0 0}$ & 1 & $\mathbf{1 0 0}$ \\
\hline
\end{tabular}

Table 9. Comparative Experimental Results of Two Algorithms (Threshold Parameter $\Delta=0.99$ in the Artificial Hypergraphs)

\begin{tabular}{|c|c|c|c|c|c|}
\hline \multicolumn{2}{|c|}{ Pairs of parameters $(n, m)$} & \multicolumn{2}{|c|}{ Algorithm 1} & \multicolumn{2}{|c|}{ Algorithm 2} \\
\hline$n$ & $\boldsymbol{m}$ & $S T$ & $\operatorname{Per}(\%)$ & ST & $\operatorname{Per}(\%)$ \\
\hline 100 & 5 & 0 & 1.06 & 0 & 1.06 \\
\hline 100 & 10 & 0 & 1.12 & 0 & 1.12 \\
\hline 100 & 20 & 0 & 1.28 & 0 & 1.28 \\
\hline 100 & 30 & 0 & 1.58 & 0 & 1.58 \\
\hline 100 & 40 & 0 & 2.4 & 0 & 2.4 \\
\hline 100 & 50 & 0 & 2.13 & 0 & 2.13 \\
\hline 100 & 60 & 0 & 2.73 & 0 & 2.73 \\
\hline 100 & 70 & 0 & 2.29 & 0 & 2.29 \\
\hline 100 & 80 & 0 & 7.74 & 0 & 7.74 \\
\hline 100 & 90 & 0 & 9.48 & 0 & 9.48 \\
\hline 100 & 800 & 0 & 98.02 & 0 & 98.02 \\
\hline 100 & 1000 & 0 & 97.03 & 0 & 97.03 \\
\hline 100 & 2000 & 0 & 100 & 0 & 100 \\
\hline 200 & 5 & 0 & 0.59 & 0 & 0.59 \\
\hline 200 & 10 & 0 & 0.64 & 0 & 0.64 \\
\hline 200 & 20 & 0 & 1.67 & 0 & 1.67 \\
\hline 200 & 30 & 0 & 1.68 & 0 & 1.68 \\
\hline 200 & 40 & 0 & 3.33 & 0 & 3.33 \\
\hline 200 & 50 & 0 & 2.06 & 0 & 2.06 \\
\hline 200 & 60 & 0 & 10.33 & 0 & 10.33 \\
\hline 200 & 70 & 0 & 10.34 & 0 & 10.34 \\
\hline 200 & 80 & 0 & 9.96 & 0 & 9.96 \\
\hline 200 & 90 & 0 & 25.97 & 0 & 25.97 \\
\hline 200 & 500 & 0 & 94.6 & 0 & 94.6 \\
\hline 200 & 800 & 0 & 100 & 0 & 100 \\
\hline 200 & 810 & 0 & 99.50 & 0 & 99.50 \\
\hline
\end{tabular}




\subsection{Analysis and Conclusions of Experimental Results}

Because parameters $n$ and $m$ in the experiments cannot be set too large due to memory constraints, we see that the time cost of Algorithm 1 and Algorithm 2 almost have no difference. From Table 8 and Table 9, we observe that the average percentage (computed by equation (8)) of Algorithm 1 is the same as Algorithm 2, which is consistent with the actual instance.

Actually, the value of each $\mid$ tree $\left(O_{i}\right) \mid(i=1,2, \ldots, n)$ of any hypergraph with unweighted hyperedges is equal to the corresponding hypergraph with weighted hyperedges. So we can obtain the same average percentage.

\section{Conclusions}

In the environment of complex Internet and Internet of Things, there is a wide variety of diverse relations among massive objects. The shortest path of a hypergraph model can characterize the associated relationship between different nodes on some degree. To search the shortest path from hypergraphs with unweighted hyperedges, this paper presents a kind of searching algorithm based on the width-first strategy. To search the shortest path from hypergraphs with weighted hyperedges, it presents a kind of searching algorithm based on the minimum-cost-first strategy. In order to enhance the reliability of the two algorithms, their correctness is proved in theory.

The next work is to do more experimental comparison and to study the relationship between the parameters and the experimental results.

\section{Acknowledgment}

This work was supported by a project (grant No. cstc2014jcyjA40035) from Chongqing Cutting-edge and Applied Foundation Research Program of China and a project (grant No. 12RC01) from Chongqing Three Gorges University of China.

\section{References}

[1] Wang, Z. P., \& Wang, Z. T. (2008). Supernetwork Theory and Its Application. Beijing: The Science Press.

[2] Voloshin, V. I. (2009). Introduction to Graph and Hypergraph Theory. New York: Nova Science Publishers.

[3] Cormen, T. H., Leiserson, C. E., Rivest, R. L., \& Stein. C. (2009). Introduction to Algorithms (3rd ed.). Massachusetts: The MIT Press.

[4] Samet, H. (2006). Foundations of Multidimensional and Metric Data Structures (3th ed.). Massachusetts: Morgan Kaufmann Publishers.

[5] Li, C. M. (1994). The algorithms of the hypergraph's shortest path. Journal of Inner Mongolia Polytechnic University, 13(1), 27-32.

[6] Qu, G., \& Ji, C. (2005). Shortest path algorithm for hypergraphs. Journal of Chongqing University (Natural Science Edition), 28(11), 106-109.

[7] Introduction of Hypergraph. Retrieved from Wikipedia website: http://en.wikipedia.org/wiki/Hypergraph

[8] Chen, X. Q. (2014). Computational model of association activity measure and its algorithmic implementation. Journal of Software, 9(5), 1135-1140.

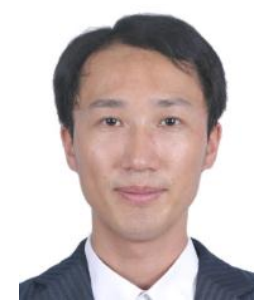

Xinquan Chen received the PhD degree from South China University of Technology in China. $\mathrm{He}$ is now an associate professor of Chongqing Three Gorges University in China and a postdoctoral researcher of University of Electronic Science \& Technologhy of China. His research interests include data mining, machine learning, clustering algorithms, and optimization methods. 